\title{
Treatment of catatonic schizophrenia and psychogenic polydipsia with clozapine:A case report
}

\begin{abstract}
Psychogenic polydipsia is a common problem in patients with chronic schizophrenia with prevalence rates varying from 5\%-10\%. No definitive treatment guidelines exist for pharmacological treatment of psychogenic polydipsia. There have been conflicting reports on antipsychotics causing and being used for treatment of polydipsia. We report a unique case of a 53 year old patient presenting with schizophrenia with catatonic features and psychogenic polydipsia treated with Clozapine. Dramatic improvement was seen not only in the polydipsia and correction of hyponatremia but also in catatonic behavior within few weeks. Clozapine may be a possible alternative option for patients presenting with catatonia and psychogenic polydipsia especially in settings where there is no availability for Electroconvulsive therapy (ECT).
\end{abstract}

Volume 2 Issue I - 2016

\author{
Inderpreet Virk, Sidiki Dabo, Christianah Y \\ Ogunlesi, Tolulope Olupona, Evaristo Akerele \\ Department of Psychiatry, Interfaith Medical Center, USA
}

Correspondence: Inderpreet Virk, Department of Psychiatry Interfaith Medical Center, I545 Atlantic Avenue, Brooklyn, NY, II2I3, USA, Tel (I) 669-226-157I, Fax 718-228-7907,

Email ivirk@interfaithmedical.com, inderpreetsvirk@yahoo.com

Received: August 30, 2016 | Published:September 20, 2016

Keywords: psychogenic polydipsia, primary polydipsia, schizophrenia, clozapine, hyponatremia, catatonia

\section{Introduction}

Schizophrenia is a chronic debilitating psychiatric disorder that affects an estimated $1 \%$ of the adult population., ${ }^{1,2}$ Psychogenic polydipsia is frequently seen in patients with chronic schizophrenia with prevalence rates varying from $5-10 \% .{ }^{1,3,4}$ It can cause dilutional hyponatremia resulting in acute brain edema. This has serious consequences which may result in delirium, seizures, coma and even death. The treatment options include various behavioral and pharmacological interventions. Due to the unclear mechanism and pathophysiology, very few controlled clinical studies have been done. ${ }^{5}$ There are few treatment options and the evidence on their efficacy is weak and conflicting. ${ }^{1,6}$ There have been conflicting reports on the use of Clozapine in patients with schizophrenia and psychogenic polydipsia. There are some reports which show that Clozapine can cause polydipsia and hyponatremia ${ }^{7}$ while other reports have shown that Clozapine can ameliorate symptoms of polydipsia. ${ }^{5,8-10}$

We report a unique case of a 53 year old Caucasian man with past history of chronic schizophrenia and multiple past psychiatric hospitalizations. Patient presented with extreme stupor, mutism and polydipsia which was successfully treated with clozapine.

\section{Clinical presentation}

Patient is a 53 year old Caucasian male with more than 30 year history of chronic schizophrenia and multiple past psychiatric hospitalizations including state hospitalizations, who were brought in to the emergency department from a residential facility due to inability to care for self and exhibiting odd and bizarre behaviour. The residential staff reported that the patient has not been eating, drinking or taking shower, socially disengaged, withdrawn and neglecting himself. Patient has been noncompliant with his medications for more than a month and slowly decompensating. On evaluation, patient was found to be in a state of stupor, mute, apathetic, aloof and listless. He actively maintained the same posture against gravity for a long duration of time.

Based on the aforementioned presentation of acute psychosis with catatonia, patient was diagnosed with schizophrenia, with catatonia ${ }^{11}$
[Diagnostic and Statistical Manual-5]. Patient was medically cleared and then transferred to the inpatient psychiatric unit for medication management and stabilization. He was started on Risperidone $2 \mathrm{mg}$ PO BID for psychosis, Ativan 1mg PO TID for catatonia and Cogentin $1 \mathrm{mg}$ PO BID for extrapyramidal symptom prevention. On the unit, patient was observed to be uncontrollably drinking excessive amounts of water and was evaluated by the medical team and neurology. After an extensive work up including Computerized Axial Tomography Scan (CAT scan) of the head to rule out other causes of polydipsia, patient was diagnosed with psychogenic polydipsia. He was placed on water restriction to $1 \mathrm{~L} /$ day and Q15 minute observation for verbal redirection in order to prevent water intoxication and hyponatremia. Patient became agitated and aggressive many times when he was redirected from drinking water from the water fountain and sink and had to be medicated often with short acting intramuscular doses of Haldol and Ativan. Haldol 2mg PO BID was added to his drug regimen on day 8 since no improvement was seen in his behavior. His catatonia responded poorly to Ativan and antipsychotics. His sodium levels were followed by the medical team due to concern of developing dilutional hyponatremia which can result in serious consequences including delirium, seizures, coma and even death. His sodium levels varied in the range of $128-131 \mathrm{mmol} / 1$. Due to the concern for developing acute brain edema due to hyponatremia patient was put on constant 1:1 observation as he required constant verbal redirection.

Due to lack of improvement seen in patient's catatonic behavior and polydipsia, on day 14 , Clozapine titration was initiated at $12.5 \mathrm{mg}$ PO BID while cross tapering of Risperidone was also initiated. Patient began to show improvement in both catatonic and polydipsic behavior in 7-8 days of starting Clozapine which was stabilized at 100mg PO BID. After 2 weeks of treatment with Clozapine patient became verbal, started interacting with staff and peers and his polydipsia resolved. His sodium levels trended upwards and were noted to return to normal limits. This effect has been sustained for 3 weeks now and patient has approached his baseline. He is compliant with his medications and weekly clozapine blood draws. He showed brighter affect with reactive mood. He became more conversational and conceptually organized with better cognitive flexibility. His activities of daily living 
and instrumental activities of daily living also significantly improved. He has returned to his baseline level of functioning.

\section{Discussion}

The pathophysiology of psychogenic polydipsia remains unclear and this limits the possibility of identifying an appropriate drug treatment 5 . Treatment becomes very difficult especially in patients with schizophrenia who are actively psychotic and polydipsic due to limited insight and lack of responsiveness to verbal redirections. Most of the cases reported in the past have been related to psychogenic polydipsia in patients with schizophrenia without catatonic features. Our case is unique as clozapine not only improved polydipsia but also improved catatonia as our patient was refractory to treatment with Ativan. In a study done by Canuso et $\mathrm{al}^{8}{ }^{8}$ on patients with polydipsic hypo-osmolemic schizophrenia, the data showed that Clozapine dose of $300 \mathrm{mg} /$ day was sufficient to normalize plasma osmolality and was well tolerated. However it is noteworthy that it was an open label study done for 24 weeks on 8 male patients only. Patients were initially given conventional neuroleptics for the first 6 weeks and then switched to Clozapine doses of $300,600,900 \mathrm{mg} /$ day depending on the tolerability. Our patient was stabilized at a dose of $200 \mathrm{mg} /$ day with sodium levels and plasma osmolality returning to normal levels. This effect has been sustained for 3 weeks now. However in future, more studies with double blind randomized controlled trials and large sample size are needed to establish the efficacy of Clozapine in patients with concurrent schizophrenia and polydipsia symptoms. Clozapine may be an alternative to ECT in patients presenting with catatonia and polydipsia especially in settings where there is no availability for ECT or patients refusing ECT.

\section{Acknowledgements}

None.

\section{Conflict of interest}

The author declares no conflict of interest.

\section{References}

1. Brookes G, Ahmed A. Pharmacological treatments for psychosisrelated polydipsia. Cochrane Database of Systematic Reviews. 2006;4:CD003544.

2. https://www.cdc.gov/mentalhealth/basics/burden.htm

3. Chen LC, Bai YM, Chang MH. Polydipsia, hyponatremia and rhabdomyolysis in schizophrenia: A case report. World J Psychiatry. 2014;4(4):150-152.

4. Victor WV, Leadbetter RA. Polydipsia-hyponatraemia syndrome: epidemiology, clinical features and treatment. CNS Drugs. 1997;7(2):121-138.

5. Diniz JB, Cordeiro Q, Zung S. Clozapine treatment for schizophreniarelated polydipsia. Rev Bras Psiquiatr. 2010;32(3):318-319.

6. Dundas B, Harris M, Narasimhan M. Psychogenic polydipsia review: etiology, differential, and treatment. Curr Psychiatry Rep. 2007;9(3):236241.

7. Ogilvie AD, Cory MF. Clozapine and hyponatremia. Lancet. 1992;340(8820):672.

8. Canuso CM, Goldman MB. Clozapine restores water balance in schizophrenic patients with Polydipsia-hyponatremia syndrome. $J$ Neuropsychiatry Clin Neurosci. 1999;11(1):86-90.

9. Leon JD, Verghese C, Stanilla JK, et al. Treatment of polydipsia and hyponatremia in psychiatric patients. Can clozapine be a new option? Neuropsychopharmacology. 1995;12(2):133-138.

10. Eleméry M, Döme P, Faludi G. Successful clozapine treatment of primary polydipsia associated with hyponatraemia in a schizophrenic patient. A case report. Neuropsychopharmacol Hung. 2007;9(4):209-213.

11. The Diagnostic and Statistical Manual of Mental Disorders DSM-5. 5th ed. American Psychiatric Association. 2013. 